\title{
Neutrophil-to-lymphocyte ratio has predictive value for lymph node involvement and distant metastasis in nasopharyngeal carcinoma patients
}

Ye Xingming ( $\nabla$ yexingming1984@126.com )

Fujian Cancer Hospital

Tianzhu Lu

Fujian Medical University

Wendong Bai

Xingjang Command General Hospital of Chinese Peoples' Liberation Army

Jing Jia

Fujian Cancer Hospital

Lin Wang

Fujian Cancer Hospital

Jingfeng Zong

Fujian cancer Hospital

Ying Chen

Fujian Cancer Hospital

Research article

Keywords: Neutrophil; NLR; nasopharyngeal carcinoma; TNM stages; metastasis; VEGF

Posted Date: July 30th, 2019

DOl: https://doi.org/10.21203/rs.2.12143/v1

License: (c) (i) This work is licensed under a Creative Commons Attribution 4.0 International License.

Read Full License 


\section{Abstract}

Background Systemic inflammatory responses play important roles in the development and progression of cancer, including the propensity for metastasis. High neutrophil-to-lymphocyte ratio (NLR) and plateletto-lymphocyte ratio (PLR) are associated with poor prognosis in nasopharyngeal carcinoma (NPC), but the underlying reasons are unclear. Here, we investigated the associations between a range of hematological parameters and T, N, and M stage in patients with NPC. Methods This was a retrospective study of 834 patients treated at our hospital for NPC between November 2011 and December 2012. A control group consisted of 647 healthy volunteers. Associations between disease stage and pretreatment laboratory data including absolute white blood cell (WBC), neutrophil, lymphocyte, and platelet counts; red cell distribution width (RDW); PLR, and NLR were analyzed using the Kruskal-Wallis test. Associations with patient survival were analyzed using the Kaplan-Meier method and log-rank test. The relationship between tumor expression of vascular-endothelial growth factor (VEGF) and circulating neutrophil counts was also evaluated in a subset of patients. Results NLR, PLR, RDW, total WBCs, neutrophils, and platelets were elevated, lymphocyte counts were lower, and hemoglobin was unchanged in NPC patients compared with healthy control subjects. NLR, PLR, and RDW were significantly associated with increasing $N$ stage $(P<0.0001, P=0.0024, P<0.0001$, respectively), and NLR, WBCs, and neutrophils were significantly associated with increasing $M$ stage $(P=0.0034, P=0.0157, P=0.0064$, respectively). Absolute neutrophil counts correlated positively with tumor VEGF expression ( $P=0.0377)$. An NLR of $\geq 2.26$ was associated with a better 5-year distant metastasis-free survival rate compared with $<2.26(89.4 \%$ vs $85.0 \%, P=0.034)$. Conclusions NLR is significantly associated with local and distant metastasis in NPC patients, and absolute neutrophil count is significantly related to tumor VEGF expression, suggesting that these parameters may be useful predictive biomarkers for NPC.

\section{Introduction}

Nasopharyngeal carcinoma (NPC) is rare worldwide but has reached epidemic proportions in Southeast Asia. In Southern China, the annual incidence of NPC is $15-30$ cases per 100,000 people [1]. Treatment of patients with NPC is based mainly on the tumor-node-metastasis (TNM) staging system. Radiotherapy alone is generally effective for early stage NPC, while chemoradiotherapy remains the standard treatment for patients with advanced disease [2]. However, even after definitive chemoradiotherapy, $15-30 \%$ of patients eventually develop distant metastases, which is the major cause of death in NPC [3,4].

Previous work has demonstrated that a high neutrophil-to-lymphocyte ratio (NLR) is associated with distant metastasis (DM) and poor treatment outcomes in patients with NPC [5-9], suggesting that NLR may represent an easily measurable and inexpensive prognostic marker in NPC. Elevated pretreatment NLR has also been associated with poor prognosis in patients with colorectal, ovarian, gastric, breast, and lung cancers [10-14]. However, the prognostic value of other blood cell parameters in metastatic NPC and the underlying changes in cell counts that drive NLR alterations remain unclear. 
In this retrospective study, we explored the relationship between immune cell counts and TNM stage in patients with NPC, with a focus on identifying potential predictors of metastasis.

\section{Methods}

\section{Patients}

The study protocol was approved by the ethics committee of Fujian Cancer Hospital, and informed consent was obtained from all patients. We reviewed the records of 834 patients treated with NPC at our hospital between November 2011 and December 2012. Pretreatment evaluations included a complete medical history, physical examination, nasopharyngoscopy examination, dental evaluation, standard laboratory tests, chest radiography or computed tomography, bone scan, and abdominal ultrasound. None of the patients had received any cancer-specific treatment at the time of data collection. Patients with evidence of infection, hematological disease, renal dysfunction, or hyperpyrexia were excluded from the study. A diagnosis of NPC was made through endoscopy-guided biopsy. Disease staging was defined according to the criteria of the 8th edition of the American Joint Committee on Cancer staging system [15]. The control group consisted of 647 healthy subjects recruited from

\section{Tissue samples and laboratory tests}

We found the information of vascular-endothelial growth factor (VEGF) expression from 834 patients. A total of 62 patients were collected tumor samples and immediately frozen in liquid nitrogen and stored at $-80^{\circ} \mathrm{C}$ until analysis, $18(29 \%)$ were determined to be positive by immunohistochemistry (score $>3$ ) or fluorescence in situ hybridization.

Laboratory data, including total white blood cell (WBC), neutrophil, lymphocyte, monocyte, and platelet counts; NLR and PLR ratios (based on counts per $\mu \mathrm{L}$ of whole blood); hemoglobin ( $\mathrm{Hb}$ ) level; and red cell width distribution (RDW), were measured as part of the automated complete blood count on a Sysmex XE-2100 analyzer (Sysmex Corporation, Japan).

\section{Statistical analysis}

Receiver operating characteristic (ROC) curves were constructed to select cut-off values for stratification of patients by pretreatment NLR. Data are expressed as the median and interquartile range (IQR) or mean and $95 \%$ confidence interval $(\mathrm{Cl})$, as appropriate. Laboratory parameters in patient and control groups were compared with $\mathrm{t}$ tests for variables with normal distribution or with the Mann-Whitney $U$ test for abnormally distributed variables. The associations between laboratory parameters and tumor stages were assessed using the Kruskal-Wallis test. DM-free survival curves were constructed using the Kaplan-Meier method and analyzed with a log-rank test. Statistical significance was defined as $P<0.05$ 
(two-tailed). All analyses were carried out using Prism 5 (GraphPad Software, La Jolla, CA, USA) and SPSS version 17 (SPSS, Chicago, IL, USA) software.

\section{Results}

\section{Patient characteristics}

Detailed information on the clinicopathological characteristics of NPC patients is shown in Table 1. A total of 834 patients were enrolled, of which 612 (73.4\%) were men. The median age at the time of surgery was 59 years (range 13 to 80 years). Evaluation of TNM stages revealed the following distribution: stage I, 0.8\% (7 patients); stage II, 5.9\% (49 patients); stage III, $49.3 \%$ (411 patients), and stage IV, 44.0\% (367 patients). Classification according to the TNM stage revealed that 261 (31.3\%) patients had deep tumor infiltration (T), 776 (93.0\%) had lymph node invasion ( $\mathrm{N}$ stage 1/2/3), and 49 (5.9\%) had DM (M1).

\section{Laboratory parameters in NPC patients and healthy control subjects}

The laboratory parameters of the 834 patients with NPC and 647 healthy subjects are shown in Table 2 . Compared with the control group, NPC patients had significantly higher total WBC, neutrophil, and platelet counts, and significantly lower lymphocyte counts. The median NLR, PLR, and RDW were also significantly higher in NPC patients compared with control subjects, but there was no difference in $\mathrm{Hb}$ levels.

\section{Association between hematologic markers and TNM stages in NPC patients}

Next, we examined the relationship between the parameters that were significantly altered in NPC patients (NLR, PLR, WBC, RDW, cell counts) and the T, N, and M stage (Fig. 1). Kruskal-Wallis analysis revealed that NLR $(P<0.0001)$, PLR $(P=0.0038)$, and RDW $(P<0.0001)$ were significantly associated with increasing $\mathrm{N}$ stage, whereas NLR and WBC were significantly higher in M1 compared with the M0 subgroup $(P=0.0034$ and $P=0.0157$, respectively) (Fig. 1). None of these parameters were significantly associated with the T stage (Fig. 1). The median neutrophil and platelet counts increased with advancing $\mathrm{N}$ stage ( $P=0.0032$ and $P=0.0164$, respectively), whereas the lymphocyte count was not associated $(P=0.1653)$ (Table 3). Neutrophil counts were significantly higher in patients with DM compared with the rest of the group (M0 vs M1, $P=0.0034$ ) (Table 3).

\section{Association between hematologic markers and overall disease stage in NPC patients}

To evaluate the relationship between the disease stage and the hematological parameters, we segregated the patients into stage I/II, III, and IV subgroups (Fig. 2). Kruskal-Wallis analysis revealed that NLR 
$(P=0.0015), \operatorname{PLR}(P=0.0008)$, and RDW $(P<0.0001)$ increased significantly with advancing disease (Fig. $2 \mathrm{~A}-\mathrm{C})$. Absolute neutrophil and platelet counts, but not lymphocyte counts, also showed significant increases in parallel with the disease stage $(P=0.0124, P<0.0001$, and $P=0.313$, respectively) (Fig. 2D $-F)$.

\section{Values of NLR, PLR, and RDW for predicting DM-free survival of NPC patients}

To assess the association with DM-free survival, we dichotomized the 834 NPC patients into groups based on NLR, PLR, RDW, and WBC cut-off values of 2.26, 87.6, 12.7, and 6.9, respectively, based on ROC curve analyses (data not shown). As shown in by the Kaplan-Meier analysis in Fig. 3, we found that patients with high baseline NLR $\geq 2.26$ had worse DM-free survival in comparison with the patients with low baseline NLR <2.26, ( $P=0.034, \mathrm{HR}: 1.5, \mathrm{Cl}$ : 1.1-1.9). However, no significant associations between DM-free survival and PLR, RDW, or WBC were detected (Fig. 3B-D).

\section{Absolute neutrophil count is positively associated with tumor VEGF expression in NPC patients}

VEGF production by tumors is known to be involved in the recruitment of pro-angiogenic neutrophils, which is associated with the development of DM $[16,17]$. Therefore, to determine whether the observed stage-dependent increase in neutrophil counts may be related to DM formation, we examined the association between neutrophils and VEGF expression in the patients. Kruskal-Wallis analysis revealed that the neutrophil count was significantly higher in patients with tumors that expressed VEGF $(P=0.0377$,

Fig. 4), suggesting that tumor VEGF production may contribute to the rise in neutrophils.

\section{Discussion}

Elevated NLR has previously been reported to be a risk factor for DM and an indicator of poor prognosis in NPC patients [5-9], although the reasons for this are obscure. In this study, we investigated a number of hematological parameters and found that several showed increases significantly associated with the $\mathrm{N}$ and $\mathrm{M}$ stage, but not the T stage. The change in NLR was driven by an increase in the absolute number of neutrophils, which correlated positively with VEGF expression in NPC tissues.

Of the parameters examined here, both NLR and PLR showed an association with advanced lymph node involvement (N3), but only NLR was significantly associated with DM and DM-free survival. This finding is consistent with other similar studies of head and neck cancers, which showed that PLR was not significantly associated with survival [18-20]. However, increasing evidence suggests that PLR is a useful prognostic factor for lung and colon cancer [21, 22]. This apparent discrepancy may be caused by tumor heterogeneity, among other factors. Taken together, our results implicate a neutrophil-dependent inflammatory response in the development of NPC metastasis.

Our finding here that absolute neutrophil counts increase with advancing NPC is consistent with previous studies showing that myeloid cells facilitate tumor advancement by promoting an inflammatory tumor microenvironment required for epithelial-mesenchymal transition, intravasation, and tumor metastasis 
[23]. Neutrophils can release growth factors such as VEGF and specific proteases [24, 25] and, consistent with this, we confirmed that VEGF expression in NPC tissues was positively associated with the neutrophil count.

We found that NPC patients had significantly lower lymphocyte counts compared with the healthy control group; however, there was no obvious association of lymphocyte count with either advancing disease stage or the development of metastases. Lymphocytes play an essential role in the anti-tumor immune response, impeding tumor cell proliferation and migration and inducing cell death [26]. Thus, although lymphocytes affect tumor growth, they likely have little influence on metastasis in NPC.

VEGF is a tumor-produced growth factor that contributes to tumor angiogenesis and metastasis [27-31], and high VEGF expression has previously been associated with poor prognosis in patients with NPC [32]. The anti-VEGF monoclonal antibody bevacizumab is currently used to treat NPC [33]; however, its precise curative effect and therapeutic index is unclear. The association between neutrophil counts and tumor VEGF expression identified in the present study suggests that these parameters could be potential evaluation markers for locally advanced and metastatic NPC.

There are several limitations to this study. First, it was retrospective. Second, the number of patients with stages I and II NPC was low (7 and 49 patients, respectively), which may have reduced the statistical power for subgroup analysis. Thus, our observations must be validated in a large-scale prospective study. Moreover, further work is required to determine whether NLR can serve as a robust predictive biomarker for NPC therapy.

In summary, we identified a stage-dependent increase in absolute neutrophil counts in patients with NPC, providing an explanation for the finding that NLR is an independent prognostic factor for DM in this patient population. Neutrophil counts also correlated positively with tumor VEGF expression, and may have utility as a prognostic marker for the development of locally advanced and metastatic NPC.

\section{Abbreviations}

AJCC: American Joint Committee on Cancer; DMFS: Distant metastasis-free survival; NLR: Neutrophil-tolymphocyte ratio; NPC: Nasopharyngeal carcinoma; OS: overall survival; ROC: Receiver operating characteristic; TNM: tumour-Node-Metastasis.

\section{Declarations}

\section{Acknowledgements}

None.

\section{Funding}


Research was supported by the Fujian Provincial Health Commission Younth Backbone Program (No.2016-ZQN-15), and the National Natural Sciences Foundation of China(No. 81802661).

\section{Authors' contributions}

XMY, TZL and WDB acquired and analyzed data. JJ and LW analyzed data. YC conceived and designed studies. JFZ wrote the manuscript. All authors read and approved the final manuscript.

\section{Ethics approval and consent to participate}

This study was conducted in compliance with institutional policy to protect patient'private information, and was approved by the Institutional Review Board of Fujian Cancer Hospital. As the current study was a retrospective assessment of routine data, the ethics committee of our Cancer Center waived the need for individual informed consent. Consent for publication not applicable.

\section{Competing interests}

The authors declare no competing financial interests.

\section{References}

1. Cao SM, Simons MJ, Qian CN. The prevalence and prevention of nasopharyngeal carcinoma in China. Chin J Cancer. 2011; 30(2): 114-119 2. Chan, AT, Chan AT, Grégoire V, Lefebvre JL, Licitra L, Felip E. Nasopharyngeal cancer: EHNS-ESMO-ESTRO Clinical Practice Guidelines for diagnosis, treatment and follow-up. Ann. Oncol. 2010; 21(Suppl 5):v187-v189. 3. Lee AW, Ng WT, Chan LL, Hung WM, Chan CC, Sze HC, Chan OS, Chang AT, Yeung RM. Evolution of treatment for nasopharyngeal cancer-success and setback in the intensity-modulated radiotherapy era. Radiother. Oncol. 2014; 110, 377-384. 4. Lai, SZ, Li WF, Chen L, Luo W, Chen YY, Liu LZ, Sun Y, Lin AH, Liu MZ, Ma J. How does intensity-modulated radiotherapy versus conventional two-dimensional radiotherapy influence the treatment results in nasopharyngeal carcinoma patients? Int J Radiat Oncol Biol Phys. 2011; 80, 661-668. 5. An X, Ding PR, Wang $\mathrm{FH}$, Jiang WQ, Li YH. Elevated neutrophil to lymphocyte ratio predicts poor prognosis in nasopharyngeal carcinoma.Tumour Biol. 2011;32(2):317-324. 6. Sun W, Zhang L, Luo M, Hu G, Mei Q, Liu D, Long G, Hu G. Pretreatment hematologic markers as prognostic factors in patients with nasopharyngeal carcinoma: neutrophil-lymphocyte ratio and platelet-lymphocyte ratio. Head Neck. 2016;38(Suppl1): E1332-E1340. 7. Chua ML, Tan SH, Kusumawidjaja G, Shwe MT, Cheah SL, Fong KW, Soong YL, Wee JT, Tan TW. Neutrophil-to-lymphocyte ratio as a prognostic marker in locally advanced nasopharyngeal carcinoma: a pooled analysis of two randomised controlled trials. Eur $\mathrm{J}$ Cancer. 2016;67:119-129. 8. Yin J, Qin Y, Luo YK, Feng M, Lang JY. Prognostic value of neutrophil-to lymphocyte ratio for nasopharyngeal carcinoma: a meta-analysis. Medicine(Baltimore). 2017; 96(29):e7577. 9. Yao J, Zhu F, Dong J, Liang Z, Yang L, Chen S, Zhang W, Lawrence WR, Zhang F, Wang S, Sun Y, Zhou G. Prognostic value of neutrophil-to-lymphocyte ratio in advanced nasopharyngeal carcinoma: a large institution-based cohort study from an endemic area. BMC Cancer. 2019;19:37-45 10. Azab B, Bhatt VR, 
Phookan J, Muruktla S, Kohn N, Terjanian T, Widmann WD. Usefulness of the neutrophil-to-lymphocyte ratio in predicting short-and long-term mortality in breast cancer patients. Ann Surg Oncol. 2012; 19:

217-224. 11. Cedres S, Torrejon D, Martinez A, Navarro A, Zamora E, Mulet-Margalef N, Felip E.

Neutrophil to lymphocyte ratio(NLR) as an indicator of poor prognosis in stage IV non-small cell lung cancer. Clin TransI Oncol. 2012;14:864-869. 12. Cho H, Hur HW, Kim SW, Kim SH, Kim JH, Kim YT, Lee K. Pre-treatment neutrophil to lymphocyte ratio is elevated in epithelial ovarian cancer and predicts survival after treatment. Cancer Immunol Immunother. 2009; 58:15-23. 13. Hung HY, Chen JS, Yeh CY, Changchien CR, Tang R, Hsieh PS, Tasi WS, You JF, You YT, Fan CW, Wang JY, Chiang JM. Effect of preoperative neutrophil-lymphocyte ratio on the surgical outcomes of stage II colon cancer patients who do not receive adjuvant chemotherapy. Int J Colorectal Dis. 2011; 26:1059-1065. 14. Jung MR, Park YK, Jeong O, Seon JW, Ryu SY, Kim DY, Kim YJ. Elevated preoperative neutrophil to lymphocyte ratio predicts poor survival following resection in late stage gastric cancer. J Surg Oncol. 2011;104:504-510 15. Wong FC, Ng AW, Lee VH, Lui CM, Yuen KK, Sze WK, Leung TW, Tung SY. Whole-field simultaneous integrated-boost intensity-modulated radiotherapy for patients with nasopharyngeal carcinoma. Int J Radiat Oncol Biol Phys. 2010; 76(1):138-145. 16. Christoffersson G, Vågesjö E, Vandooren J, Lidén M, Massena S, Reinert RB, Brissova M, Powers AC, Opdenakker G, Phillipson M. VEGF-A recruits a proangiogenic MMP-9-delivering neutrophil subset that induces angiogenesis in transplanted hypoxic tissue. Blood. 2012;120(23):4653-4662. 17. Segawa Y, Oda Y, Yamamoto H, Shiratsuchi H, Hirakawa N, Komune S, Iwamoto Y, Oda Y. Close correlation between CXCR4 and VEGF expression and their prognostic implications in nasopharyngeal carcinoma. Oncol Rep. 2009; 21:1197-1202. 18. Young CA, Murray LJ, Karakaya E, Thygesen HH, Sen M, Prestwich RJ. The prognostic role of the neutrophil-to-lymphocyte ratio in oropharyngeal carcinoma treated with chemoradiotherapy. Clin Med Insights Oncol. 2014; 8:81-86. 19. Farhan-Alanie OM, McMahon J, McMillan DC. Systemic inflammatory response and survival in patients undergoing curative resection of oral squamous cell carcinoma. $\mathrm{Br} J$ Oral Maxillofac Surg. 2015; 53:126-131. 20. Selzer E, Grah A, Heiduschka G, Kornek G, Thurnher D. Primary radiotherapy or postoperative radiotherapy in patients with head and neck cancer: Comparative analysis of inflammation-based prognostic scoring systems. Strahlenther Onkol. 2015; 191:486-494 21. Ding N, Pang ZF, Shen H, Ni Y, Du J, Liu Q. The prognostic value of PLR in lung cancer, a Meta-analysis Based on Results from a Large Consecutive Cohort. Sci Rep. 2016; 6:34823. 22. Liu Y, Chen Y, Chen S, Li Z, Zhao X, Zhang W, Zhi F. Platelet to lymphocyte ratio might be a prognostic factor of colorectal cancer (CRC): A meta-analysis. Int J Clin Exp Med. 2016; 9:11090-11095 23. Elinav E, Nowarski R, Thaiss CA, Hu B, Jin C, Flavell RA. Inflammation-induced cancer: crosstalk between tumours, immune cells and microorganisms. Nat Rev Cancer. 2013;13:759-771. 24. Paramanathan A, Saxena A, Morris DL. A systematic review and meta-analysis on the impact of pre-operative neutrophil lymphocyte ratio on long term outcomes after curative intent resection of solid tumours. Surg Oncol. 2014; 23(1):31-39 25. Kusumanto YH, Dam WA, Hospers GA, Meijer C, Mulder NH. Platelets and granulocytes, in particular the neutrophils, form important compartments for circulating vascular endothelial growth factor. Angiogenesis. 2003; 6(4):283-287. 26. Swann JB, Smyth MJ. Immune surveillance of tumours. J Clin Invest. 2007; 117(5):1137-1146 27. Fujita K, Sano D, Kimura M, Yamashita Y, Kawakami M, Ishiguro Y, Nishimura G, Matsuda $\mathrm{H}$ and Tsukuda M: Anti tumour effects of bevacizumab in combination with 
paclitaxel on head and neck squamous cell carcinoma. Oncol Rep. 2007;18: 47-51. 28. Vaklavas C, Lenihan D, Kurzrock R and Tsimberidou AM. Anti-vascular endothelial growth factor therapies and cardiovas-cular toxicity: What are the important clinical markers to target? Oncologist. 2010; 15: 130-141. 29. Li Y, Lu J, Zhou S, Wang W, Tan G, Zhang Z, Dong Z, Kang T ,Tang F. Clusterin induced by $\mathrm{N}, \mathrm{N}^{\prime}$ ' Dinitrosopiperazine is involved in nasopharyngeal carcinoma metastasis. Oncotarget. 2016; 7: 5548-5563. 30. Chen HH, Weng BQ, Cheng KJ, Liu HY, Wang SQ and Lu YY. Effect of the vascular endothelial growth factor expression level on angiopoietin-2-mediated nasopharyngeal carcinoma growth. Vasc Cell. 2014; 6:4. 31. Grunstein J, Roberts WG, Mathieu Costello O, Hanahan D and Johnson RS: tumour-derived expression of vascular endothelial growth factor is a critical factor in tumour expansion and vascular function. Cancer Res. 1999; 59: 1592-1598. 32. Li YH, Hu CF, Shao Q, Huang MY, Hou JH, Xie D, Zeng YX and Shao JY: Elevated expressions of survivin and VEGF protein are strong independent predictors of survival in advanced nasopharyngeal carcinoma. J Transl Med. 2008; 6: 1. 33. Lee NY, Zhang Q, Pfister DG, Kim J, Garden AS, Mechalakos J, Hu K, Le QT, Colevas, AD, Glisson BS, Chan AT, Ang KK. Addition of bevacizumab to standard chemoradiation for locoregionally advanced nasopharyngeal carcinoma (RTOG 0615): a phase 2 multi-institutional trial. Lancet Oncol. 2012; 13(2):172-180.

\section{Figures}

A

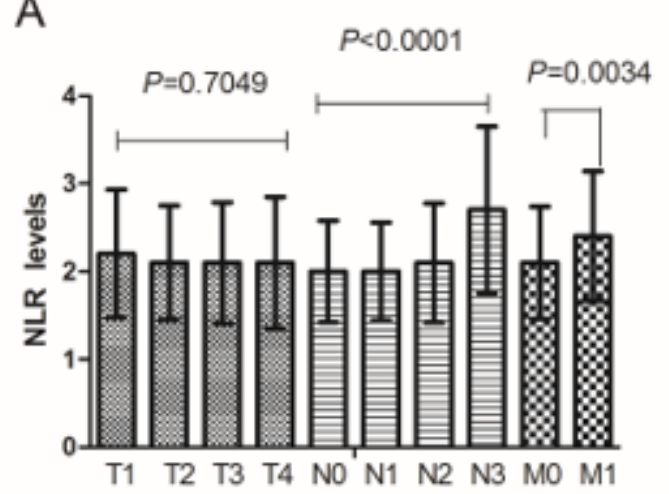

C

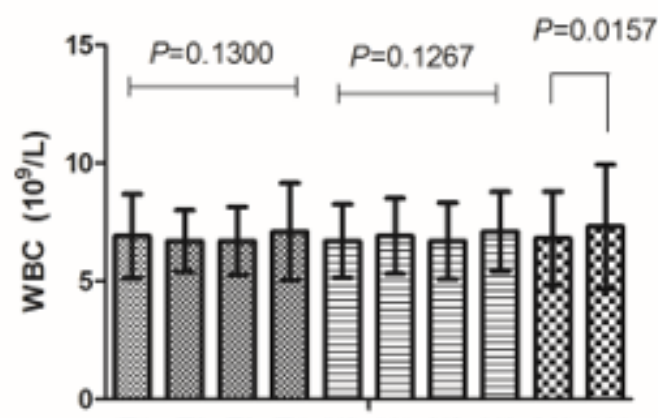

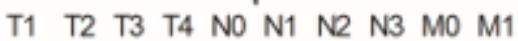
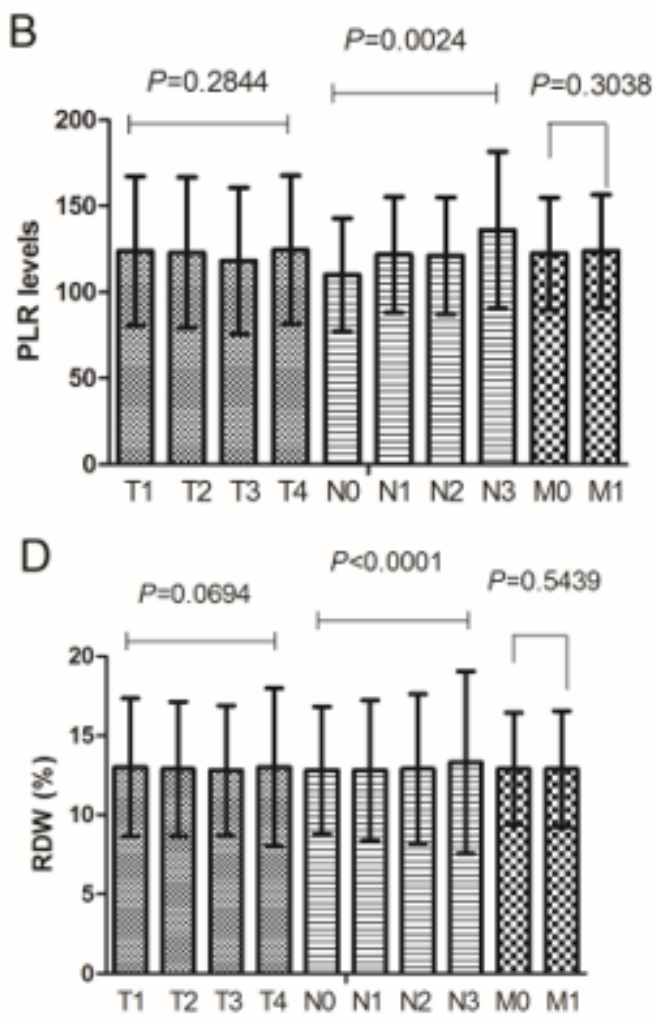

Figure 1 
Analysis of blood parameters in NPC patients according to T, N, and M stages. (a) NLR, (b) PLR, (c) WBC, and (d) RDW in 834 NPC patients grouped by T, N, and M stages.

A

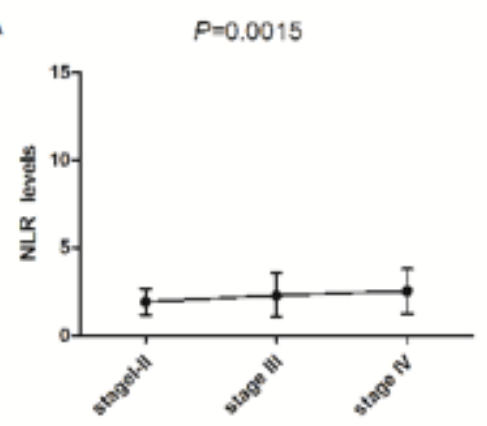

D

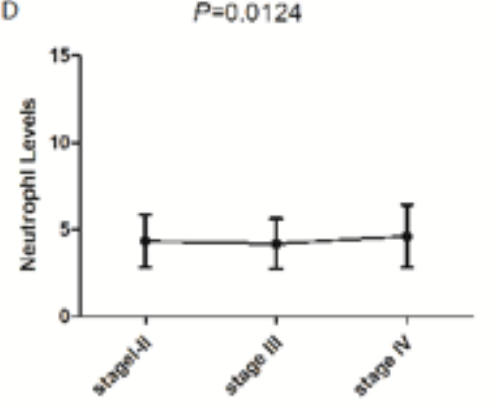

B

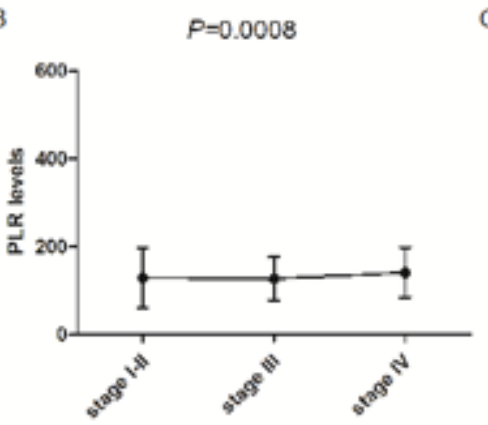

E

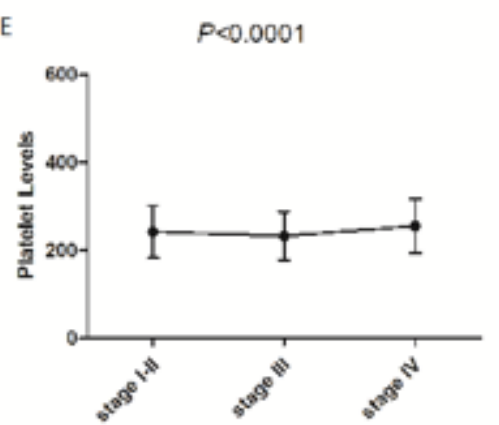

c
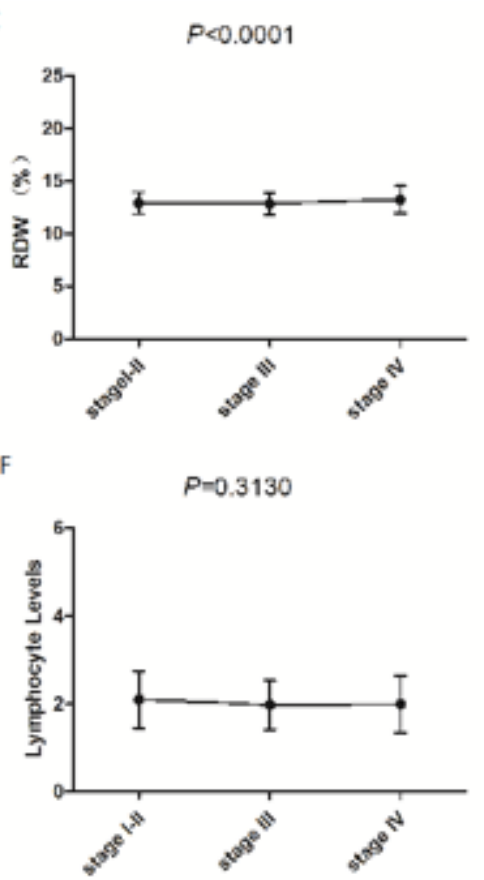

Figure 2

Analysis of blood parameters in NPC patients according to overall disease stage. (a) NLR, (b) PLR, (c) RDW, (d) neutrophil count, (e) platelet count, and (f) lymphocyte count in 834 NPC patients grouped by stage I/II, III, and IV disease. Cell counts are expressed as the number $\times 109 / \mathrm{L}$. 

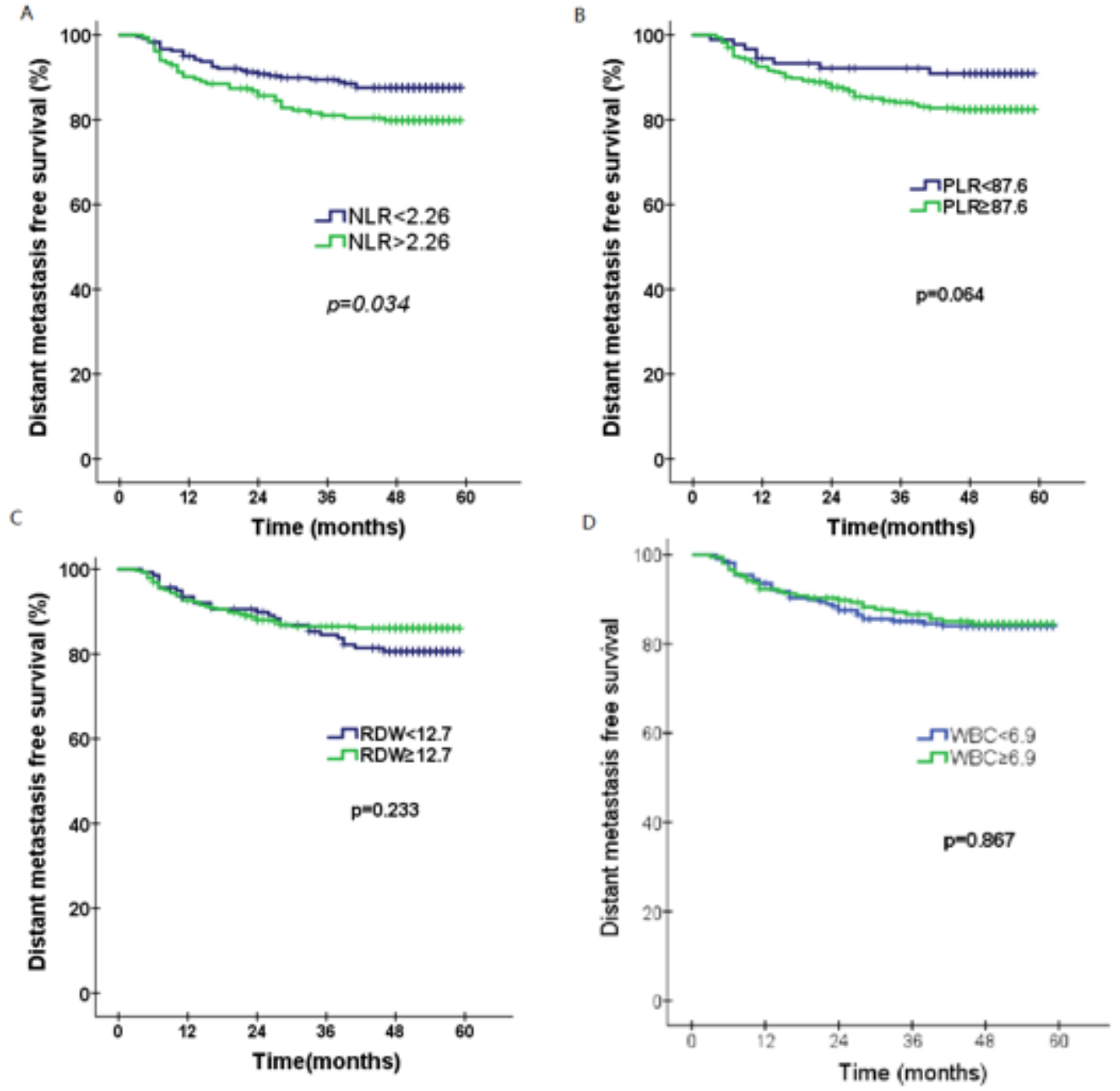

\section{Figure 3}

Kaplan-Meier DM-free survival curves for NPC patients stratified by hematological parameters. Patients were assigned to two groups based on cut-off values for (a) NLR, (b) PLR, (c) RDW, and (d) WBC, as determined by ROC curve analysis. Group sizes for the $\geq$ and < cut-off values, respectively, were (a) $\geq 2.26$ and $<2.26$, (b) $\geq 87.6$ and $<87.6$ (c) $\geq 12.7$ and $<12.7$, and (d) $\geq 6.9$ and $<6.9$. 


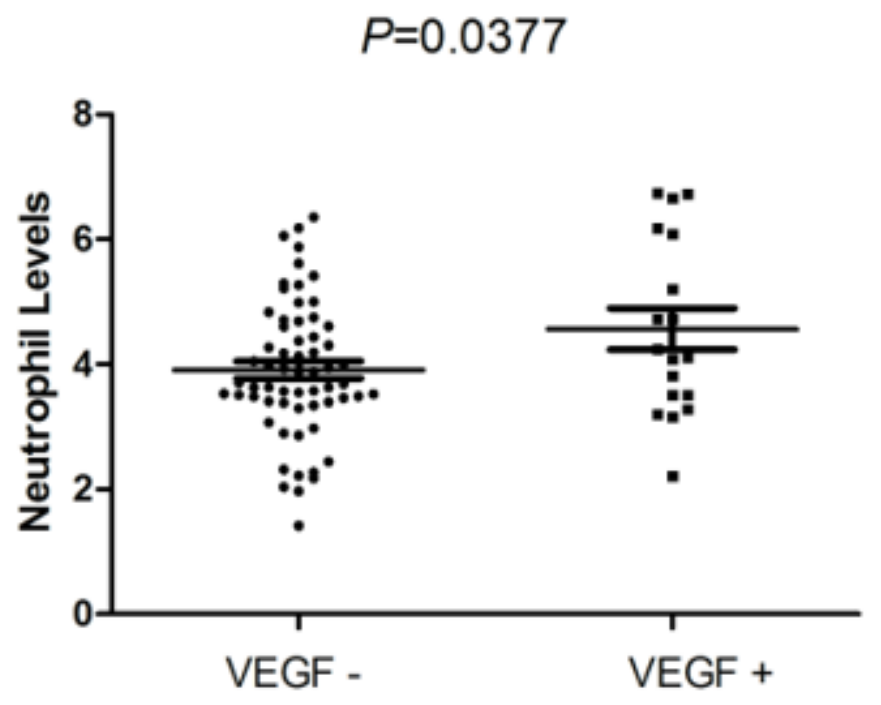

Figure 4

Correlation between the neutrophil count and tumor VEGF expression in NPC patients. NPC tissue samples from 62 patients were analyzed for VEGF mRNA by fluorescence in situ hybridization or immunohistochemistry. Neutrophils are expressed as the number $\times 109 / \mathrm{L}$. $P=0.0377$.

\section{Supplementary Files}

This is a list of supplementary files associated with this preprint. Click to download.

- supplement1.pdf

- supplement2.pdf

- supplement3.pdf 